\title{
A NOTE ON THE DISINTEGRATION OF MEASURES
}

\author{
S. K. BERBERIAN
}

ABSTRACT. Complements to a theorem of Bourbaki on the disintegration of measures on separated topological spaces.

If $X$ and $T$ are separated topological spaces, $\nu$ is a measure on $X$, and $p$ is a mapping of $X$ into $T$, then, under suitable hypotheses, there exist a measure $\mu=p(\nu)$ on $T$ and a family $\left(\lambda_{t}\right)_{t \in T}$ of measures on $X$ indexed by $T$, such that

(a) for every $t \in T, \lambda_{t}$ is carried by $p^{-1}(\{t\})$, and

(b) $\nu=\int \lambda_{t} d \mu(t)$ in an appropriate sense; moreover,

(c) $\left\|\lambda_{t}\right\|=1$ for locally $\mu$-almost every $t$ in $T$, and

(d) the family $\left(\lambda_{t}\right)_{t \in T}$ is determined by properties (a) and (b) up to a locally $\mu$-negligible set [2, 2 , No. 7, Proposition 13].

The purpose of this note is to repair a gap in the proofs of (c) and (d) given in [2]. \{To pinpoint the problem: in the proof of (c) on p. 42 of [2], the application of (12) is unjustified since the function $f \phi_{A}$ need not be universally measurable.\} For greater clarity, we shall operate under somewhat weaker hypotheses than [2] (to which we refer for notations and terminology).

For the rest of the paper, $X$ and $T$ denote separated topological spaces, $\nu$ is a measure on $X$ (all measures under discussion are positive), $\mu$ is a measure on $T$, and $t \mapsto \lambda_{t}$ is a mapping of $T$ into the set of measures on $X$. If $f \in \mathscr{F}_{+}(X)$ (that is, $f$ is a positive numerical function on $X$ ), we define positive numerical functions $h_{f}$ and $h_{f}^{*}$ on $T$ by the formulas $h_{f}(t)=\lambda_{t}(f)$, $h_{f}^{*}(t)=\lambda_{t}^{*}(f)$ (thus $\left.0 \leqslant h_{f} \leqslant h_{f}^{*}\right)$. We assume that the mapping $t \mapsto \lambda_{t}$ satisfies the following condition (obviously weaker than the condition (b) of [2, §2, No. 7, Proposition 13]):

(*) If $f \in \mathscr{F}_{+}(X)$ is lower semicontinuous or upper semicontinuous, then $h_{f}$ is $\mu$-measurable and $\mu^{\circ}\left(h_{f}\right)=\nu^{\cdot}(f)$.

In more florid notation, the formula in (*) means that

$$
\int_{X}^{\cdot} f(x) d \nu(x)=\int_{T}^{\cdot} d \mu(t) \int_{X}^{\cdot} f(x) d \lambda_{t}(x) .
$$

LEMMA 1. For every $f \in \mathscr{F}_{+}(X)$, one has $\nu^{*}(f) \geqslant \mu^{*}\left(h_{f}^{*}\right) \geqslant \mu^{*}\left(h_{f}\right)$.

Proof. Formally the same as in [1, §3, No. 2, Proposition 3].

LEMMA 2. If $f \in \mathscr{F}_{+}(X)$ is $\nu$-negligible, then $f$ is $\lambda_{t}$-negligible for locally $\mu$-almost every $t$ in $T$.

Received by the editors July 15, 1977.

AMS (MOS) subject classifications (1970). Primary 28A50. 
Proof. Immediate from Lemma 1 (cf. [1, §3, No. 2, Corollary 1 of Proposition 3]).

LEMMA 3. If $f \in \mathscr{F}_{+}(X)$ is $\nu$-moderated, then $f$ is $\lambda_{t}$-moderated for locally $\mu$-almost every $t$ in $T$.

Proof. Write $f=\sum_{n \geqslant 1} f_{n}$, where $f_{1}$ is $\nu$-negligible and, for each $n \geqslant 2, f_{n}$ vanishes outside a compact set $[2, \S 1$, No. 9, Corollary 3 of Proposition 14]; the proof is then formally the same as $[1, \S 3$, No. 2, Corollary 2 of Proposition 3].

Assertions (ii) and (iii) of the following proposition fill the gap mentioned at the beginning of the paper (cf. [2, §2, No. 7, Remark 2]):

Proposition. Assuming condition (*) is verified, if $\nu$ is moderated and $f \in \mathscr{F}_{+}(X)$ is $\nu$-measurable, then (i) $f$ is $\lambda_{t}$-measurable and $\lambda_{t}$-moderated for locally $\mu$-almost every $t$ in $T$, (ii) the function $h_{f}: t \mapsto \lambda_{t}^{*}(f)$ is $\mu$-measurable, and (iii) $\mu^{\circ}\left(h_{f}\right)=\nu^{\circ}(f)$.

Proof. (cf. [1, §3, No. 2, Proposition 5]). Let $\left(X_{n}\right)_{n>1}$ be a denumerable $\nu$-crushing of $X$ such that the restriction of $f$ to each of the compact sets $X_{n}$ is continuous [2, $\S 1$, No. 8, Propositions 10, 11].

(i) In view of Lemma 3 , we need only show that $f$ is $\lambda_{t}$-measurable for locally $\mu$-almost every $t$. The proof is formally the same as in $[1, \S 3$, No. 2 , Proposition 4a)].

(ii), (iii) Let $N=X-\cup X_{n}$; thus $\nu^{*}(N)=\nu^{\cdot}(N)=0$. Write $f_{0}=f \varphi_{N}$ and $f_{n}=f \varphi_{X_{n}}$ for $n \geqslant 1$. The conclusions (ii) and (iii) hold for $f_{0}$ by Lemma 2. For $n \geqslant 1, f_{n}$ is upper semicontinuous, hence conditions (ii) and (iii) hold for $f_{n}$ by the hypothesis (*). Let $S$ be the set of $t \in T$ such that $f_{0}$ is not $\lambda_{t}$-negligible; by Lemma $2, \mu^{\circ}(S)=0$. For $n \geqslant 1, f_{n}$ is universally measurable. It follows that if $t \in T-S$, then $f_{n}$ is $\lambda_{t}$-measurable for all $n \geqslant 0$, hence so is $f$, whence $h_{f}(t)=\Sigma_{n} h_{f_{n}}(t)\left[2, \S 1\right.$, No. 5, Proposition 4]. Thus $h_{f}=\Sigma_{n} h_{f_{n}}$ locally $\mu$-almost everywhere, therefore $h_{f}$ is also $\mu$-measurable and $\mu\left(h_{f}\right)=\Sigma_{n} \mu\left(h_{f_{n}}\right)=$ $\Sigma_{n} \nu^{\circ}\left(f_{n}\right)=\nu^{\bullet}(f)$ by $[2, \S 1$, No. 5, Proposition 4].

COROllary. Assuming condition (*) is verified, if $\nu$ is moderated and $f \geqslant 0$ is $\nu$-integrable, then $h_{f}$ is essentially $\mu$-integrable, $f$ is $\lambda_{t}$-integrable for locally $\mu$-almost every $t$ in $T$, and $\nu^{\circ}(f)=\mu^{\circ}\left(h_{f}\right)$, that is,

$$
\int f(x) d \nu(x)=\int d \mu(t) \int f(x) d \lambda_{t}(x)
$$

\section{REFERENCES}

1. N. Bourbaki, Eléments de mathématique. Fasc. XXI. Libre VI: Intégration. Chap. V, 2ième ed., Actualités Sci. Indust., no. 1244, Hermann, Paris, 1967. MR 35 \# 322.

2. __ Éléments de mathématique. Fasc. XXXV. Livre VI: Intégration. Chap. IX, Actualités Sci. Indust., no. 1343, Hermann, Paris, 1969. MR 43 \#2183.

Department of Mathematics, University of TeXas, Austin, TeXas 78712 